\title{
The impact of 27-hydroxycholesterol on endometrial cancer proliferation
}

\author{
Douglas A Gibson, Frances Collins, Fiona L Cousins*, Arantza Esnal Zufiaurre and Philippa T K Saunders \\ Medical Research Council Centre for Inflammation Research, The University of Edinburgh, Queen's Medical Research Institute, Edinburgh, UK \\ Correspondence should be addressed to D A Gibson: d.a.gibson@ed.ac.uk \\ *(F L Cousins is now at The Ritchie Centre, Hudson Institute of Medical Research, Clayton, Victoria, Australia)
}

\begin{abstract}
Endometrial cancer (EC) is the most common gynaecological malignancy. Obesity is a major risk factor for EC and is associated with elevated cholesterol. 27-hydroxycholesterol $(27 \mathrm{HC})$ is a cholesterol metabolite that functions as an endogenous agonist for Liver $X$ receptor (LXR) and a selective oestrogen receptor modulator (SERM). Exposure to oestrogenic ligands increases risk of developing EC; however, the impact of $27 \mathrm{HC}$ on EC is unknown. Samples of stage 1 EC $(n=126)$ were collected from postmenopausal women undergoing hysterectomy. Expression of LXRs (NR1H3, LXR $\alpha$; NR1H2, LXR $\beta$ ) and enzymes required for the synthesis (CYP27A1) or breakdown (CYP7B1) of 27HC were detected in all grades of EC. Cell lines originating from well-, moderate- and poorly-differentiated ECs (Ishikawa, RL95, MFE 280 respectively) were used to assess the impact of $27 \mathrm{HC}$ or the LXR agonist GW3965 on proliferation or expression of a luciferase reporter gene under the control of LXR- or ER-dependent promoters (LXRE, ERE). Incubation with 27HC or GW3965 increased transcription via LXRE in Ishikawa, RL95 and MFE 280 cells $(P<0.01)$. 27HC selectively activated ER-dependent transcription $(P<0.001)$ in Ishikawa cells and promoted proliferation of both Ishikawa and RL95 cells $(P<0.001)$. In MFE 280 cells, $27 \mathrm{HC}$ did not alter proliferation but selective targeting of LXR with GW3965 significantly reduced cell proliferation $(P<0.0001)$. These novel results suggest that $27 \mathrm{HC}$ can contribute to risk of EC by promoting proliferation of endometrial cancer epithelial cells and highlight LXR as a potential therapeutic target in the treatment of advanced disease.
\end{abstract} Key Words

\section{Introduction}

Endometrial cancer (EC) is the most common gynaecological malignancy and the fourth most common cancer in women in developed countries with incidence increasing in line with rising rates of obesity (reviewed in Onstad et al. 2016). Obesity is a major modifiable risk factor for EC and is thought to contribute to increased risk of malignancy in part due to increased exposure to estrogens, which enhance the risk of aberrant proliferation within the endometrium (Sanderson et al. 2017). Obesity is also associated with an adverse metabolic profile, which is postulated to independently increase risk of EC (Trabert et al. 2015).

A recent meta-analysis supported a positive association between dietary cholesterol consumption and endometrial cancer risk (Gong et al. 2016). Notably, obesity also puts individuals at risk of developing an adverse, raised, cholesterol profile. Cholesterol metabolites such as the oxysterol 27-hydroxycholesterol (27HC) have been (c) 2018 The authors Published by Bioscientifica Ltd. Printed in Great Britain

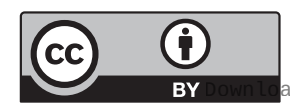

This work is licensed under a Creative Commons Attribution 4.0 International License. 
demonstrated to promote cancer growth and metastasis in studies on breast cancer (Nelson et al. 2013, Wu et al. 2013), providing a plausible mechanistic link between increased adiposity and EC risk. 27HC is a primary metabolite of cholesterol, synthesised by the action of sterol 27-hydroxylase (CYP27A1) and metabolised by 25-hydroxycholesterol 7- $\alpha$-hydroxylase (CYP7B1; (1)). 27HC acts as an endogenous agonist for the Liver $\mathrm{X}$ receptor (LXR), a ligand-activated transcription factor involved in the regulation of cholesterol homeostasis. Two isoforms of LXR have been identified; LXR $\alpha$ (encoded by $N R 1 H 3)$, which is predominantly expressed in the liver, kidney and small intestine but exhibits low expression in other tissues, and LXR $\beta$ (encoded by NR1H2), which is ubiquitously expressed. Based largely on studies in breast cancer, LXRs have been proposed as a novel anti-cancer target and the LXR-selective agonists GW3965 and T0901317 are reported to decrease proliferation of LXR-expressing breast cancer cell lines (MCF7, T47D, MDA-MB231) as well as the prostate cancer cell line LNCaP (Vedin et al. 2009, Kim et al. 2010). To the best of our knowledge, LXR expression has not been reported in human EC tissues and the impact of either $27 \mathrm{HC}$ or LXR agonists on the endometrium or endometrial malignancies is not known.

In addition to activating LXRs, 27HC can also bind oestrogen receptors (ER) (Umetani et al. 2007) and acts as an endogenous selective oestrogen receptor modulator (SERM) (DuSell et al. 2008). 27HC has diverse impacts and its SERM activity is reported to be both tissue selective and context dependent. For example, 27HC acts as a competitive antagonist of ERs expressed in the vasculature and can antagonise E2-mediated endothelial cell migration and re-endothelialisation (Umetani et al. 2007). In contrast, in the absence of $\mathrm{E} 2,27 \mathrm{HC}$ is reported to act as an agonist to ER $\alpha$ (ESR1) to increase cell adhesion and expression of pro-inflammatory cytokines such as tumour necrosis factor alpha (TNFA) and interleukin 6 (IL6) (Umetani et al. 2014) by endothelial cells and macrophages. Notably, 27HC is also reported to increase proliferation of ER $\alpha$-positive breast cancer cell lines and promotes MCF7 tumour xenograft growth in mice by stimulating ER-dependent cell proliferation ( $\mathrm{Wu}$ et al. 2013). Given selective LXR agonists have anti-proliferative effects (Vedin et al. 2009), these studies suggest that proliferative effects of $27 \mathrm{HC}$ may be mediated via ER and that relative expression of LXR or ER isoforms may define the impact of the ligand.

ERisoformsareexpressedinECtissuesandERexpression changes with disease progression (Collins et al. 2009).
We have previously reported that ERo is readily detectable in both epithelial and stromal cells in well-differentiated cancers but is significantly reduced in poorly differentiated cancers. In contrast, expression of ESR2 variants (ER $\beta 1$, $2,5)$ was readily detected in well, moderate and poorly differentiated stage $1 \mathrm{ECs}$ (Collins et al. 2009). We therefore postulated that 27HC might have distinct effects in EC depending on the bioavailability of ER isoforms present at different stages of disease progression.

Obesity and the metabolic syndrome are both associated with an increased risk of developing pre-malignant and malignant endometrial disease (Sanderson et al. 2017) but whether the cholesterol metabolite $27 \mathrm{HC}$ has an impact on EC risk/progression is not known. In the current study, we assessed the expression of the enzymes required for synthesis (CYP27A1) and breakdown (CYP7B1) of 27HC and assessed expression of the cognate receptors LXR $\alpha$ and LXR $\beta$ in primary human stage I endometrial adenocarcinomas $(n=126)$ and postmenopausal endometrial controls $(n=9)$. The impact of 27HC and the LXR-selective agonist GW3965 on EREand LXRE-dependent expression of a reporter gene, as well as cellular proliferation, was assessed in three EC cell lines which phenocopy well-, moderate- and poorly differentiated stage I ECs. Our novel findings demonstrate that 27HC can alter responses in EC cells and highlight LXR as a potential therapeutic target. Taken together, our findings suggest increased exposure to $27 \mathrm{HC}$ may increase risk of development and progression of EC.

\section{Materials and methods}

\section{Human tissue samples}

Endometrial adenocarcinoma tissue was collected from postmenopausal women undergoing total abdominal hysterectomy who had been previously diagnosed to have endometrioid adenocarcinoma of the endometrium; they had received no treatment before surgery (Supplementary Table 1 , see section on supplementary data given at the end of this article). Written informed consent was obtained from all subjects prior to surgery, and ethical approval was granted by the Lothian Research Ethics Committee (LREC1999/6/4). Methods were carried out in accordance with NHS Lothian Tissue Governance guidelines. All ECs were confined to the uterus (International Federation of Obstetrics and Gynaecology, FIGO, stage 1 as described in Collins et al. 2009). Diagnosis of adenocarcinoma was confirmed histologically by an experienced gynaecological pathologist, and tissues were further graded as well

This work is licensed under a Creative Commons Attribution 4.0 International License. 
differentiated (G1), moderately differentiated (G2) or poorly differentiated (G3). Samples were anonymised and patient follow-up information was not available. However, survival statistics for stage $1 \mathrm{EC}$ in the UK are reported as 99\% 1-year survival and 95.3\% 5-year survival (Cancer Research UK; http://www.cancerresearchuk.org/ health-professional/cancer-statistics/statistics-by-cancertype/uterine-cancer - accessed November 2017) and Information Services Division Scotland figures, which cover the stage $1 \mathrm{EC}$ samples collected in the current study, report 92.9\% 1-year survival and 83.2\% 5-year survival for all uterine cancers (http://www.isdscotland. org/Health-Topics/Cancer/Cancer-Statistics/FemaleGenital-Organ/\#uterus - accessed November 2017).

Postmenopausal controls $(n=9)$ were obtained from women undergoing surgery for non-malignant gynaecological conditions. None of the women were receiving hormonal therapy. A total of 126 EC tissue samples were analysed; 3 samples per grade were assessed for immunohistochemistry and $n=30$ well-differentiated cancers, $n=64$ moderately differentiated and $n=32$ poorly differentiated samples were assessed for qPCR studies. A minimum of 10 samples at each grade were analysed for each gene, detailed sample numbers are included in Supplementary Table 2 . Tissue for immunohistochemistry was collected in neutral buffered formalin (NBF), RNA extraction samples were collected in RNALater (Qiagen).

\section{Measurement of mRNA}

Isolation of mRNAs, preparation of cDNAs and analysis by qPCR were performed according to standard protocols (Bombail et al. 2010); samples were quantified by relative standard curve method or by the comparative $\Delta \Delta \mathrm{Ct}$ method with $C Y C$ as internal control. Primers/probes are given in Supplementary Table 3.

\section{Immunohistochemistry}

Single antibody immunohistochemistry using 3,3'-diaminobenzidine tetra-hydrochloride (DAB) detection was performed as described previously (Collins et al. 2009). Double immunofluorescence was carried out with antibodies directed against LXR or ER $\alpha$ and the proliferation marker Ki67. Details of antibodies and dilutions are provided in Supplementary Table 4. Primary antibodies were incubated at $4^{\circ} \mathrm{C}$ overnight. Antigen detection was performed using Tyramide signal amplification (Perkin Elmer) system followed by nuclear counterstaining with DAPI (4',6-diamidino- 2-phenyl-indole dihydrochloride). Negative controls were incubated in the absence of primary antibody but otherwise processed as above; no staining was detected in no primary controls for any of the antibodies used (not shown). Images were captured using a LSM 710 Confocal microscope (Zeiss) at $\times 40$ magnification.

\section{Cell cultures}

Threeendometrialadenocarcinoma cell lines representative of well-, moderately- or poorly differentiated cancers were used. Ishikawa cells were obtained from the European Collection of Cell Culture (ECACC no. 99040201, Wiltshire, UK). This cell line was originally derived from a well-differentiated adenocarcinoma of a 39-year-old woman (Nishida et al. 1985) and reported to express both ER $\alpha$ and ER $\beta$ protein (Johnson et al. 2007). RL95-2 cells (ATCC CRL-1671; hereafter RL95) were originally derived from a Grade 2 moderately differentiated endometrial adenocarcinoma (Way et al. 1983) and reported to express both ER $\alpha$ and ER $\beta$ protein (Yang et al. 2008, Li et al. 2014). MFE-280 (ECACC no. 98050131) were derived from a recurrent, poorly differentiated, endometrial adenocarcinoma and have low/undetectable expression of $\mathrm{ER} \alpha$ and $\mathrm{ER} \beta$. Cells were maintained in DMEM/F12 (Sigma) supplemented with 10\% FBS, $100 \mathrm{U}$ penicillin, streptomycin and $0.25 \mu \mathrm{g} / \mathrm{mL}$ fungizone (Invitrogen) at $37^{\circ} \mathrm{C}$ in $5 \% \mathrm{CO}_{2}$. Media for RL95 was supplemented with $0.005 \mathrm{mg} / \mathrm{mL}$ insulin (Sigma). Cells were incubated with 27-hydroxycholesterol (27HC; Tocris Cat. No. 3907) using stocks diluted in ethanol to give final concentrations ranging from $10^{-5} \mathrm{M}$ to $10^{-8} \mathrm{M}$ or GW 3965 hydrochloride (GW; Tocris Cat. No. 2474) using stocks diluted in DMSO to give final concentrations ranging from $10^{-5} \mathrm{M}$ to $10^{-8} \mathrm{M}$. Some cultures were co-incubated with the antiestrogen fulvestrant (ICI 182,780; Tocris Cat. No. 1047) diluted in DMSO at a final concentration of $10^{-6} \mathrm{M}$. Appropriate vehicle control incubations were included in all studies. All cell lines were authenticated using the Promega PowerPlex 21 system (Eurofins Genomics, Ebersberg, Germany).

\section{Reporter assays}

An adenoviral vector containing a $3 \times$ ERE-tk-luciferase reporter gene was prepared as described previously (Collins et al. 2009). Cells were cultured in DMEM without phenol red and containing charcoal stripped foetal calf serum (CSFCS) for $24 \mathrm{~h}$ before being infected with Ad-ERE-Luc at a MOI of 25. Activation of

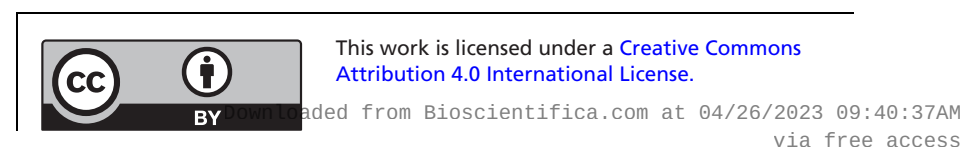


LXR-dependent signal transduction was assessed according to manufacturer's instructions using reagents from the Cignal LXR Reporter Kit, which includes positive and negative controls as well as a luciferase reporter gene under the control of tandem repeats of the LXR transcriptional response element (LXRE) (Qiagen, CCS-0041L).

Cells were treated for $24 \mathrm{~h}$ and luciferase activities were determined using 'Bright Glo' reagents (Promega). Luminescence was measured using Fluostar Microplate Reader (BMG labtech) and fold-change in luciferase activity was calculated relative to vehicle control for each treatment.

\section{Proliferation assays}

The impact of treatments on cell proliferation was assessed using CyQUANT Direct Cell Proliferation Assay (Thermo Fisher, C35011) according to manufacturer's instructions and nuclear fluorescence measured using Novostar Microplate Reader (BMG labtech). For each cell line investigated, cell number was quantified using a standard curve of known cell numbers and fold-change in cell number calculated relative to vehicle control for each treatment.

\section{Statistical analysis}

Statistical analysis was performed using GraphPad prism. One-way ANOVA was used to determine significance between treatments in data that were normally distributed. Non-parametric testing was utilised where sample sizes were insufficient to confirm normality of data distribution; Kruskal-Wallis test was used to assess differences between treatments. Where data were analysed as fold-change, significance was tested using one sample $t$ test and a theoretical mean of 1 . Criterion for significance was $P<0.05$. All data are presented as mean \pm S.E.M.

\section{Results}

\section{Enzymes that regulate bioavailability of 27-hydroxycholesterol and its cognate receptor LXR are expressed in EC}

Messenger RNAs encoded by CYP7B1 and CYP27A1 were detected in all cancer grades (Fig. 1A and B); expression of $C Y P 7 B 1$ was significantly lower in poorly differentiated cancers compared to moderately differentiated cancers $(P<0.05)$. Relative expression of CYP27A1 tended to be higher in poorly differentiated cancers, but this was not significant. We next assessed relative expression of mRNAs encoding the LXR receptors known to bind 27HC: NR1H3 (LXR $\alpha)$ and NR1H2 (LXR $\beta)$ were detected in all cancer grades (Fig. 1C and D). Expression of NR1H3 was significantly lower in moderately differentiated cancers compared to postmenopausal controls $(P<0.01)$. Expression of NR1H2 did not change between sample groups.

\section{Immunolocalisation of LXR and the proliferation marker Ki67 in EC tissue sections}

The expression of LXR in EC tissue sections was assessed by immunohistochemistry using an antibody that detected
A

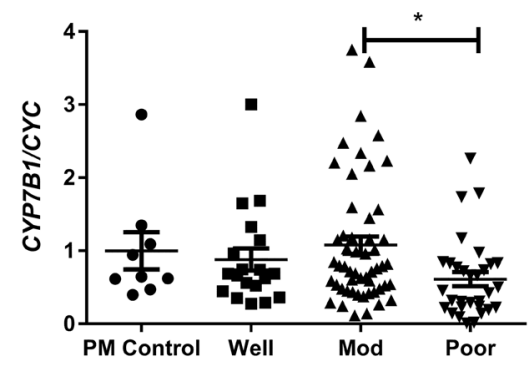

C

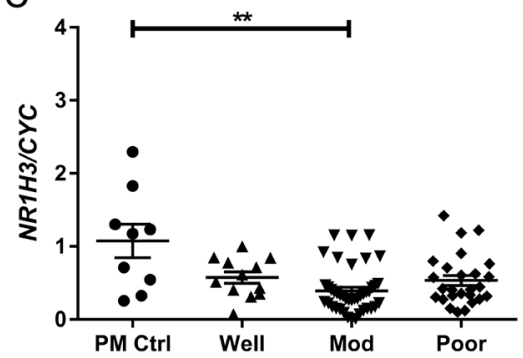

B

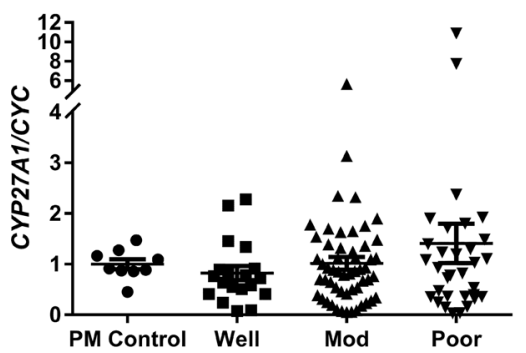

D

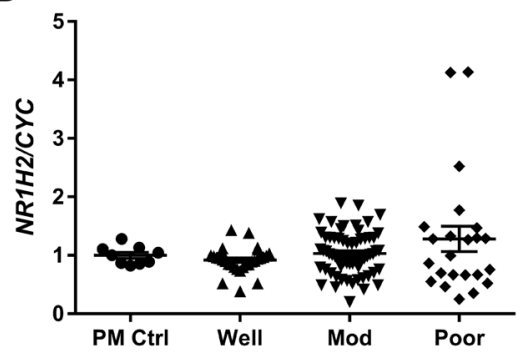

Figure 1

27HC signalling pathway is expressed in endometrial cancer and altered with disease severity. The expression of CYP7B1, CYP27A1, NR1H3 (LXR $\alpha)$ and NR1H2 (LXRß) relative to internal control gene $C Y C$ was assessed by $q P C R$ in postmenopausal control endometrium (PM Ctrl) and in endometrial cancer tissue homogenates from well-, moderately- and poorly differentiated endometrial adenocarcinomas. Relative expression of mRNAs encoding CYP7B1 (A) were decreased in poorly differentiated cancers compared to moderately differentiated cancers but CYP27A1 was not significantly different (B). Relative expression of mRNAs encoding NR1H3 (C; LXR $\alpha$ ) were significantly decreased in moderately differentiated cancers compared to postmenopausal control tissues whilst NR1H2 (LXR $\beta)$ was not significantly different (D). $* P<0.05, * * P<0.01$. Kruskal-Wallis test with multiple comparisons. PM, $n=9$; Well $n=12-30$; Mod, $n=42-64$; Poor, $n=23-32$. All data are presented as mean \pm S.E.M. http://erc.endocrinology-journals.org https://doi.org/10.1530/ERC-17-0449
() 2018 The authors Published by Bioscientifica Ltd. Printed in Great Britain
This work is licensed under a Creative Commons Attribution 4.0 International License. 
both isoforms of LXR (mouse anti-LXR; sc-271064). LXR was readily detected in well-, moderately- or poorly differentiated cancers and was immunolocalised to both stromal and epithelial cells (Supplementary Fig. 1). To assess if LXR expression was associated with cell proliferation within EC tissue, we performed double immunofluorescence staining for both LXR and the proliferation marker Ki67 (Fig. 2). In well-differentiated cancers (Fig. 2A), nuclear immunoexpression of Ki67 (red staining) was detected which co-localised (yellow arrows) with LXR expression (green staining, note that single channel views show that the intensity of LXR staining varied between cells). Whilst careful evaluation of single channel views confirmed that the majority of LXR-positive cells were also immunopositive for Ki67 some cells were Ki67 negative (white arrows). In contrast, in moderately differentiated cancers (Fig. 2B), both markers were detected but few cells appeared to co-localise (yellow arrows) although LXR-positive cells (white arrows) were found in close association with proliferating cells. In poorly differentiated cancers (Fig. 2C), few cells expressed both markers. Ki67positive cells were clustered in regions with limited LXR expression and no co-expression of LXR and Ki67 was detected. LXR+Ki67- cells (white arrows) were detected close to $\mathrm{Ki}^{+}{ }^{+}$cells. We also assessed the expression of $\mathrm{ER} \alpha$ and Ki67 in EC tissues (Supplementary Fig. 2) as this receptor is implicated in the regulation of proliferation in normal endometrium (Lubahn et al. 1993, Frasor et al. 2003). Consistent with our previous study, ER $\alpha$ was not detected in the poorly differentiated cancers (Collins et al. 2009) and immunoexpression of Ki67 was clearly independent of $\mathrm{ER} \alpha$ with an increase in abundance of positive nuclei in poor (sample codes 910/2178) as compared to well or moderately differentiated tissue where co-localisation of $\mathrm{ER} \alpha$ and $\mathrm{Ki} 67$ was readily detected.

\section{HC activates LXRE- and ERE-dependent transcription in endometrial epithelial cancer cells and alters proliferation of EC cells}

Having demonstrated expression of enzymes and receptors required for $27 \mathrm{HC}$ signalling, we extended our observational study by exploring the impact of the ligand on endometrial epithelial cancer cell lines chosen to model well-, moderately- or poorly differentiated stage I cancers; Ishikawa, RL95 and MFE 280. Protein expression of both LXR isoforms was confirmed by western blot in all cell lines studied (Supplementary Fig. 3A and B).
We assessed the mRNA expression of LXRs in these cell lines and found that their expression phenocopied that found in tissue samples (Supplementary Fig. 3). NR1H3 mRNA expression was significantly decreased in RL95 (moderately differentiated) cells compared to MFE 280 (poorly differentiated; $P<0.01$; Supplementary Fig. 3C). Consistent with tissue mRNA expression patterns, NR1H2 was not different between cell lines (Supplementary Fig. 3D). Messenger RNAs encoded by both ER genes; ER $\alpha$ (ESR1) and ER $\beta$ (ESR2; ER $\beta 1$ specific primers) were detected in all of the cell lines (Supplementary Fig. 4). ESR1 mRNAs were significantly reduced in RL95 and MFE280 compared to Ishikawa cells (Supplementary Fig. 4A) consistent with patterns of expression in intact tissue (Supplementary Fig. 2). ESR2 mRNA was significantly reduced in MFE280 cells compared to Ishikawa (Supplementary Fig. 4B). As $27 \mathrm{HC}$ is both an endogenous agonist for LXR and a SERM, the impact of 27HC on LXRE- and ERE-dependent transcription was investigated in the EC cell lines. 27HC significantly increased LXRE-dependent transcription in a dose-dependent manner in all 3 cell lines and was maximally stimulated by $10^{-5} \mathrm{M} 27 \mathrm{HC}$ (Fig. 3A, B and C). In contrast, $27 \mathrm{HC}$ only stimulated ERE-dependent transcription in Ishikawa cells (Fig. 3D) at $10^{-8} \mathrm{M}(P<0.01)$ and $10^{-7} \mathrm{M}(P<0.0001)$. The impact of $27 \mathrm{HC}$ was abrogated by co-incubation with the anti-oestrogen fulvestrant (ICI 182,780) consistent with ER dependence. In contrast to Ishikawa cells, 27HC had little impact on ERE-dependent transcription in RL95 (Fig. 3E) and MFE280 cells (Fig. 3F). As 27HC could activate both ERE- and LXRE-promoters, we assessed its impact on cell proliferation (Fig. 3G, H and I). 27HC induced proliferation of Ishikawa cells at concentrations ranging from $10^{-8} \mathrm{M}$ to $10^{-6} \mathrm{M}(P<0.01)$, but this was inhibited at the highest concentration $\left(10^{-5} \mathrm{M}\right.$, $P<0.0001)$. 27HC significantly increased proliferation in RL95 cells at concentrations of $10^{-7} \mathrm{M}(P<0.001)$ or greater. In contrast, $27 \mathrm{HC}$ did not alter proliferation of MFE 280 cells at any of the concentrations investigated. Neither RL95 nor MFE280 cell lines expressed CYP7B1 (Supplementary Fig. 3E and F) precluding the potential for in vitro metabolism limiting cell responses to $27 \mathrm{HC}$ in these cell lines.

\section{Targeting LXR with the synthetic agonist GW3965 activates LXRE-dependent transcription and alters cell proliferation in a cell-specific manner}

Incubation of cells with the LXR-selective agonist GW3965 significantly increased LXRE-dependent transcription in a dose-dependent manner (Fig. 4) consistent with 

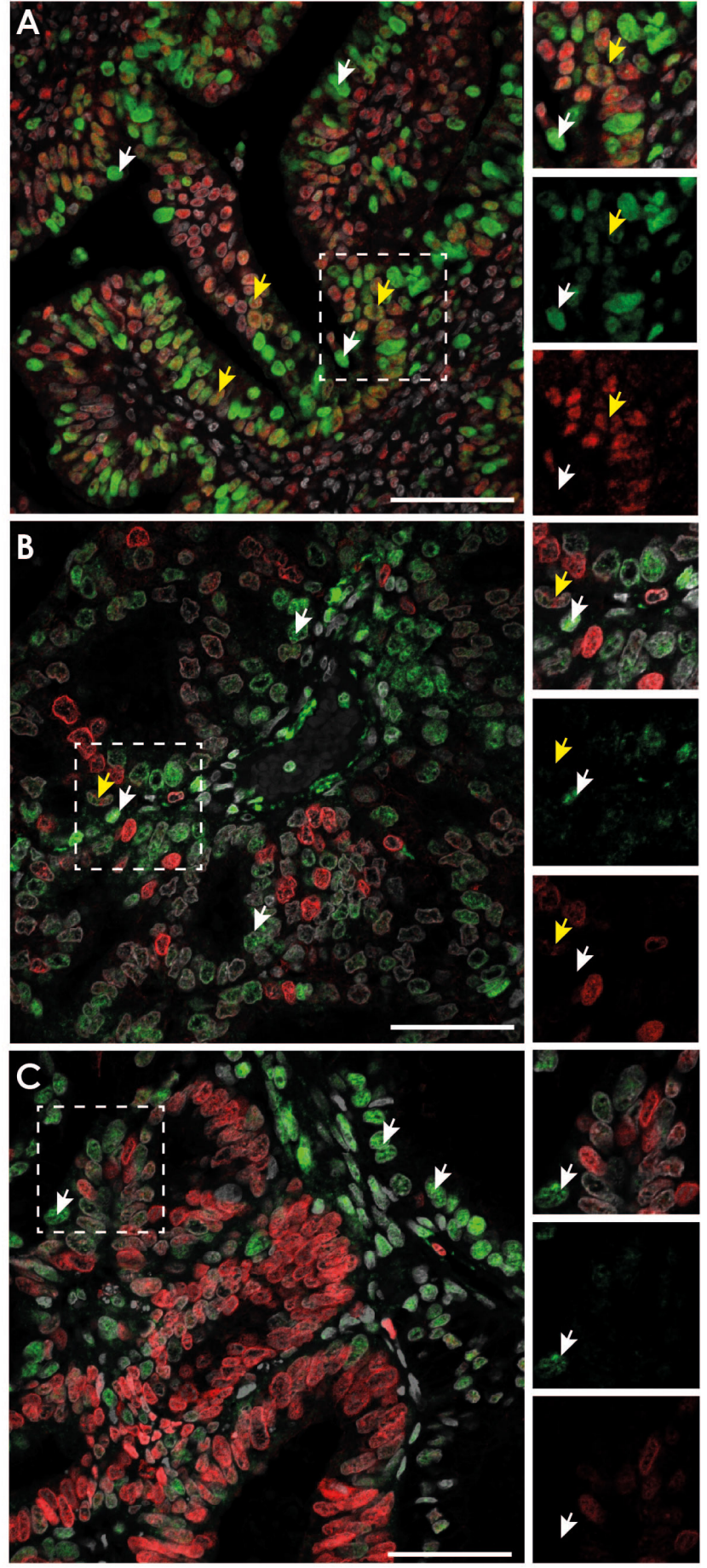

LXR Ki67 DAPI

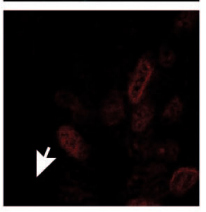

Figure 2

Expression of LXR and the proliferation marker Ki67 in endometrial cancer. The expression of LXR (antibody identified both isoforms) and the proliferation marker Ki67 was assessed by immunohistochemistry in endometrial cancer tissue sections. In well-differentiated cancers (A), LXR was expressed throughout the tissue and localised to the nuclei of both stromal and epithelial cells (green staining). Nuclear immunoexpression of Ki67 (red staining) was detected and co-localised with LXR expression (yellow arrows) although some LXR-positive cells did not co-express Ki67 (white arrows). In moderately differentiated cancers (B) both markers expression of LXRs in the EC cell lines (Supplementary Fig. 3). In contrast to 27HC, GW3965 significantly and robustly increased LXRE-dependent transcription at concentrations $\geq 10^{-8} \mathrm{M}$ in Ishikawa (Fig. 4A) and RL95 (Fig. 4B) and $\geq 10^{-7} \mathrm{M}$ in MFE280 cells (Fig. 4C). Although LXR reporter responses were similar in the different cell lines, proliferation responses were strikingly different. In Ishikawa cells, treatment with GW3965 at concentrations $10^{-8} \mathrm{M} \quad(P<0.01)$ and $10^{-5} \mathrm{M} \quad(P<0.01) \quad$ significantly increased proliferation (Fig. 4D). In contrast, GW3965 significantly and robustly decreased cell proliferation at all concentrations investigated in both RL95 (Fig. 4E) and MFE 280 cells (Fig. 4F).

\section{Discussion}

To date, no study has assessed the association between the cholesterol metabolite $27 \mathrm{HC}$ and EC. EC incidence rates have increased by $\sim 50 \%$ since the early 1990 s and approximately $57 \%$ of endometrial cancers in the United States have been attributed to being overweight or obese (Cancer Research, UK; http://www.cancerresearchuk. org - accessed November 2017, and Calle \& Kaaks 2004). Although increased exposure to adipose-derived estrogens is believed to increase aberrant proliferation within the endometrium (Zhao et al. 2016), recent evidence supports an independent role for obesityassociated metabolic factors in modulating EC risk. Notably, both elevated triglycerides and increased dietary cholesterol consumption are reported to be associated with increased EC risk (Lindemann et al. 2009, Gong et al. 2016). Importantly, concentrations of the cholesterol metabolite $27 \mathrm{HC}$ are increased in postmenopausal women (Burkard et al. 2007) and are associated with increased risk of breast cancer. Several studies have identified that $27 \mathrm{HC}$ has an adverse impact on breast cancer (Nelson et al. 2013, Wu et al. 2013) but whether $27 \mathrm{HC}$ can affect EC has not been investigated previously.

In light of these studies, we hypothesised that 27HC signalling could contribute to the aetiology of endometrial cancer and influence disease progression, and we investigated this using both archival human tissue as well as cell lines

were detected but did not appear to co-localise; only few cells expressed both LXR and Ki67 (yellow arrows). Most LXR-positive cells did not co-express Ki67 (white arrows). This was also true of poorly differentiated cancers (C), few cells expressed both LXR and Ki67 (yellow arrows) although LXR-positive cells were found in close association with proliferating cells (white arrows). Images representative of at least 3 different patients per cancer grade. Nuclear counterstain DAPI (grey). All scale bars $50 \mu \mathrm{M}$. 
A

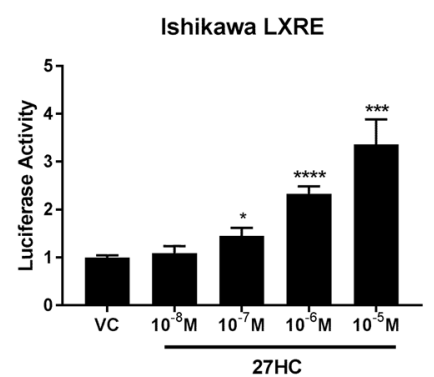

D

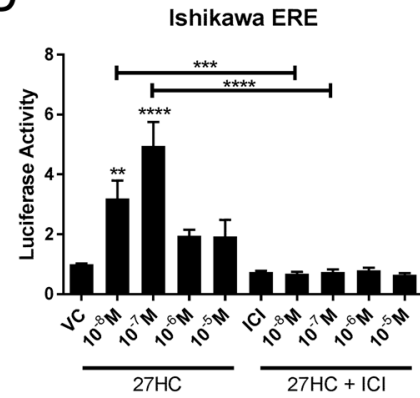

G

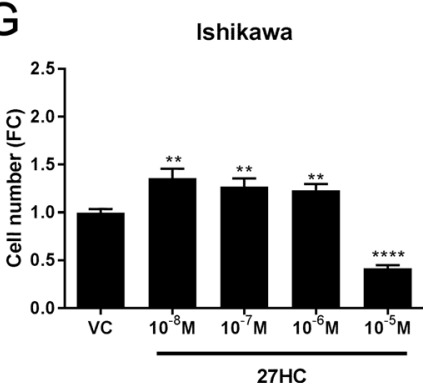

B

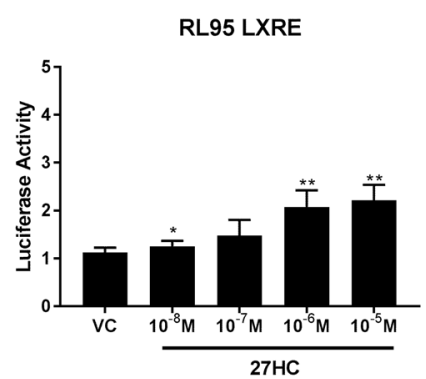

E

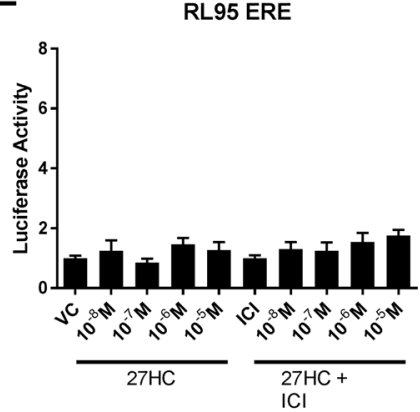

$\mathrm{H}$

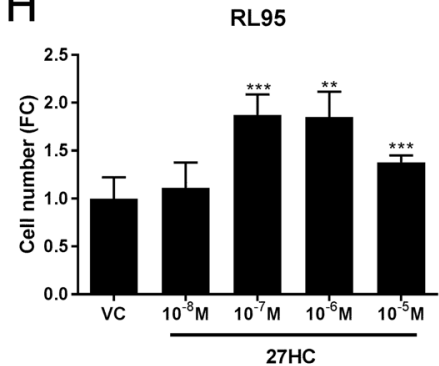

C

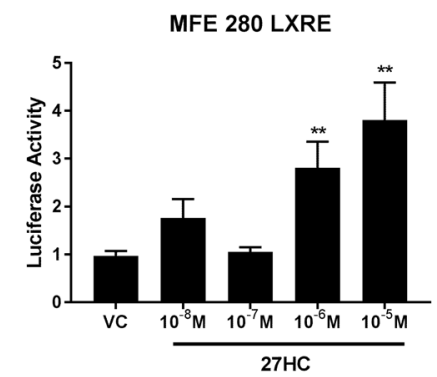

F

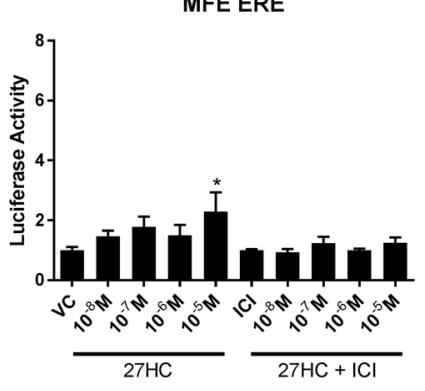

I

MFE 280

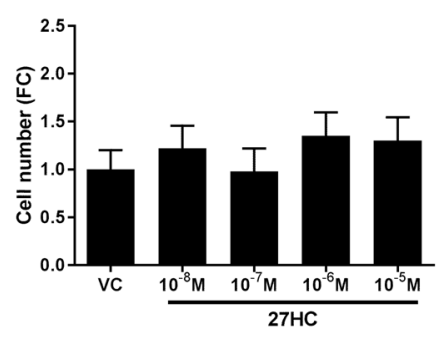

Figure 3

27HC activates LXRE- and ERE-dependent transcription in endometrial epithelial cancer cells and alters proliferation. The cholesterol metabolite 27-hydoxycholesterol (27HC) is the endogenous agonist for LXR and is also classified as selective oestrogen receptor modulator. The impact of 27HC on LXRE- (A, B and C) and ERE-dependent (D, E and F) transcription was investigated by luciferase reporter assay in endometrial cancer cell lines; Ishikawa, RL95 and MFE280. 27HC significantly increased LXRE-dependent transcription in a dose-dependent manner in each endometrial cancer cell line. 27HC stimulated ERE-dependent transcription only at lower concentrations and was significantly increased by $10^{-8} \mathrm{M} 27 \mathrm{HC}(P<0.01)$ and maximally stimulated by $10^{-7} \mathrm{M} 27 \mathrm{HC}(P<0.0001)$. The $27 \mathrm{HC}$ effect was abrogated by co-incubation with the antiestrgoen Fulvestrant $(\mathrm{ICI} 182,780 ; \mathrm{ICI})$ at all concentrations of 27HC (D). 27HC did not increase ERE-dependent transcription in RL95 (E) and was only increased by $10^{-5} \mathrm{M} 27 \mathrm{HC}(P<0.05)$ in MFE280 cells (F). Cell proliferation was assessed by CyQuant direct proliferation assay in each cell line $(\mathrm{G}, \mathrm{H}$ and $\mathrm{I})$. Proliferation of Ishikawa cells was increased by $10^{-8} \mathrm{M}$ $(P<0.01), 10^{-7} \mathrm{M}(P<0.01)$ and $10^{-6} \mathrm{M}(P<0.01) 27 \mathrm{HC}$ but decreased by $10^{-5} \mathrm{M} 27 \mathrm{HC}(P<0.0001 ; \mathrm{G})$. Proliferation of RL95 cells was increased by $10^{-7} \mathrm{M}$ $(P<0.001), 10^{-6} \mathrm{M}(P<0.01)$ and $10^{-5} \mathrm{M}(P<0.001) 27 \mathrm{HC}(\mathrm{H}) .27 \mathrm{HC}$ did not affect proliferation in MFE280 cells $(\mathrm{I}) . * P<0.05, * * P<0.01, * * * P<0.001$,

$* * * * P<0.0001$. One sample $t$ test and a theoretical mean of 1. All data are presented as mean \pm S.E.M.

that are derived from different grades of EC. We obtained new evidence for expression of the enzymes required for the both the synthesis (CYP27A1) and breakdown (CYP7B1) of 27HC. As concentrations of CYP7B1 mRNAs were significantly decreased in poorly compared to moderately differentiated cancers and expression of CYP27A1 did not change significantly across EC grades; we believe this would favour increased bioavailability of $27 \mathrm{HC}$ with increasing grade. These findings appear to parallel those reported for
ER+ breast cancer where decreased expression of CYP7B1 and increased CYP27A1 has been reported in tumours compared to normal breast tissues ( $\mathrm{Wu}$ et al. 2013). Furthermore, we found that the endogenous receptor for 27HC, LXR, was immunolocalised to stage 1 cancers and was expressed throughout the tissue and localised to the nuclei of both stromal and epithelial cells.

We sought to establish if 27HC could alter responses in EC cells by acting via its cognate receptor, LXR, or 
A

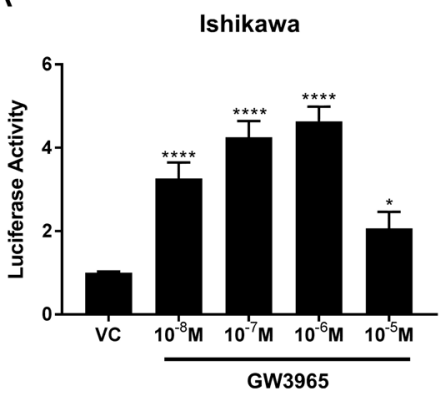

D

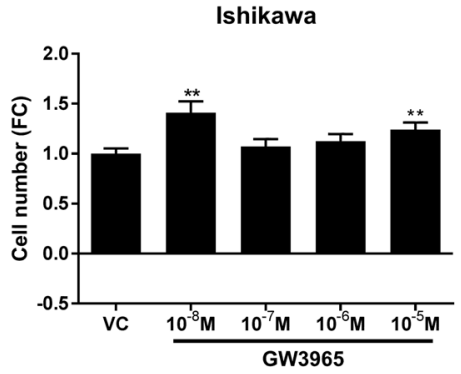

B

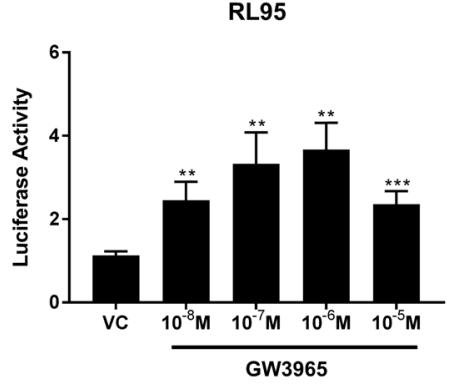

E

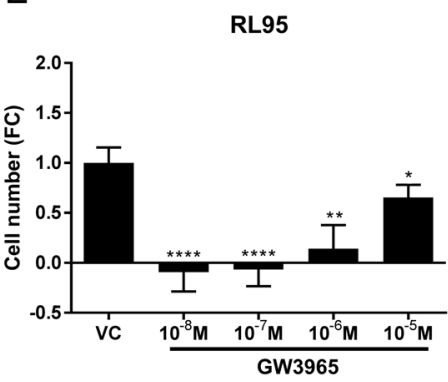

C

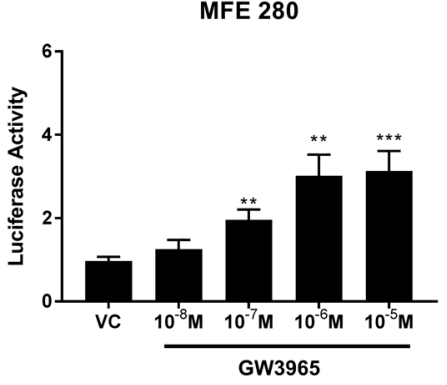

F

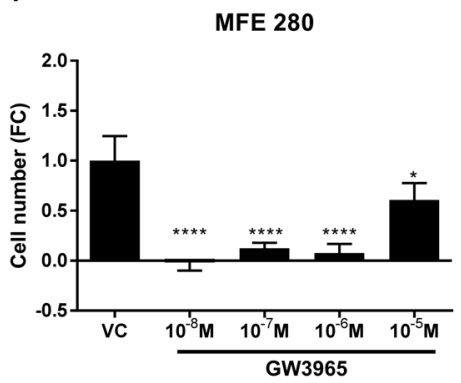

Figure 4

LXR agonist GW3965 activates LXRE-dependent transcription and alters proliferation in endometrial epithelial cancer cells. The impact of the LXR synthetic agonist GW3965 on LXRE-dependent transcription (A, B and C) and on cell proliferation (D, E and F) was assessed in endometrial cancer cell lines; Ishikawa, RL95 and MFE280. GW3965 significantly increased LXRE-dependent transcription in a dose-dependent manner in each endometrial cancer cell line. GW3965 significantly increased LXRE-dependent transcription at all concentrations assessed and was maximally increased by $10^{-6} \mathrm{M}$ GW3965 in Ishikawa cells $(P<0.0001)(A)$ and RL95 cells $(P<0.01)(B)$. LXRE-dependent transcription and was not increased by $10^{-8} \mathrm{M}$ GW3965 but maximally increased by $10^{-5} \mathrm{M} 27 \mathrm{HC}(P<0.001)$ in MFE280 cells (C). Cell proliferation was assessed by CyQuant direct proliferation assay in each cell line $(\mathrm{D}, \mathrm{E}$ and $\mathrm{F})$. Proliferation of Ishikawa cells was increased by $10^{-8} \mathrm{M}(P<0.01)$ and by $10^{-5} \mathrm{M} 27 \mathrm{HC}(P<0.01)$ (D). In contrast, proliferation of RL95 cells was decreased by $10^{-8} \mathrm{M}(P<0.001), 10^{-7} \mathrm{M}(P<0.001), 10^{-6} \mathrm{M}(P<0.01)$ and $10^{-5} \mathrm{M}(P<0.05) \mathrm{GW} 3965$ (E). Proliferation of MFE280 cells was decreased by $10^{-8} \mathrm{M}(P<0.001), 10^{-7} \mathrm{M}(P<0.0001), 10^{-6} \mathrm{M}(P<0.0001)$ and 10-5 M $(P<0.05) \mathrm{GW} 3965(\mathrm{I}) . * P<0.05, * * P<0.01, * * * P<0.001, * * * * P<0.0001$. One sample $t$ test and a theoretical mean of 1 . All data are presented as mean \pm S.E.M.

via estrogen receptors, which are known to regulate endometrial proliferation. 27HC activated LXR-dependent transcription in all cell lines tested. In contrast, we found that 27HC activated ERE-dependent reporter gene expression in well-differentiated cancer cells (Ishikawa; $\mathrm{ER} \alpha+\mathrm{ER} \beta+$ ) but not in those from moderately (RL95; ER $\alpha^{\text {lowER }} \beta+$ ) or poorly differentiated cancers (MFE280; ER $\alpha^{\text {lowER }} \beta^{\text {low })}$. However, 27HC increased proliferation of both Ishikawa and RL95 cells but not MFE280 cells consistent with reported ER expression in these cell lines (Johnson et al. 2007, Yang et al. 2008, Li et al. 2014). Our immunohistochemistry analysis (Supplementary Fig. 2) supported these in vitro findings. We found that the proliferation marker Ki67 co-localised with ER $\alpha$ in well- and moderately differentiated cancers consistent with a key role for this receptor in mediating endometrial epithelial cell proliferation (Lubahn et al. 1993, Frasor et al. 2003). In poorly differentiated cancers, ER $\alpha$ was not detected consistent with previous reports (Collins et al. 2009). It has been reported that $27 \mathrm{HC}$, acting as a SERM, can impact on ER $\alpha$ - or ER $\beta 1$-dependent regulation of cell function (He \& Nelson 2017) and the oestrogenic effects of $27 \mathrm{HC}$ could therefore be mediated via either ER isoform in EC cells. In endometrial endothelial cells, which express ER $\beta$ but not ER $\alpha$, oestrogenic effects are mediated via ER $\beta$ tethered to Sp1 and not via direct binding to ERE (Greaves et al. 2013). Furthermore, it has been reported that 27HC promotes proliferation of ER $\alpha$-positive LNCaP prostate cancer cells via ER $\beta$ (Lau et al. 2000, Raza et al. 2017) which may account for the apparent discrepancy between ERE reporter assay and cell proliferation responses in RL95 cells observed in the current study. Taken together, these findings reveal the potential for 27HC generated within the EC tissue microenvironment to influence ER-dependent transcription and proliferation via ERs expressed in early-grade stage $1 \mathrm{EC}$.

Although the association between ERs and endometrial proliferation is well recognised, there is limited data investigating the role of LXR in this process. Expression of LXR $\alpha$ and LXR $\beta$ mRNA has been previously reported in endometrium and myometrium of mice (Mouzat et al. 2007) and 27HC is reported 
to increase mouse uterine weight, consistent with an uterotrophic action; however, whether this was mediated via ER or LXR was not investigated (Wu et al. 2013). In mice, targeted ablation of the receptor subtypes revealed that $L x r \alpha-/-$ but not $L x r \beta-/-$ females had reduced endometrial areas compared to wildtype mice consistent with a role for $\mathrm{LXR} \alpha$ in promoting endometrial growth/proliferation in that species (Mouzat et al. 2007). In the current study, we found that LXR co-localised with the proliferation marker Ki67 in well-differentiated but not moderate- or poorly differentiated EC tissues. In vitro assays verified this finding as the synthetic LXR agonist GW3965 had a cell-selective impact on the EC cell lines. In Ishikawa cells GW3965 increased proliferation, whereas in RL95 and MFE280 cells equimolar concentrations of agonist blocked proliferation. Given that LXR expression was detected in all grades of EC, this may suggest LXR could be an effective therapeutic target in some ECs, albeit in a grade-dependent context. Indeed, GW3965 is reported to abrogate E2-mediated increases in MCF7 breast cancer cell proliferation and has been proposed as an anti-proliferative ligand in this context (Vedin et al. 2009).

LXR classically acts as a heterodimeric partner of retinoid $X$ receptor (RXR). RXR is expressed in the nuclei of endometrial epithelial cells throughout the menstrual cycle (Fukunaka et al. 2001) as well as in EC tissues (Nickkho-Amiry et al. 2012). Interestingly, LXR-RXR functions as a 'permissive' heterodimer and binding of either an LXR agonist or the RXR agonist 9-cis retinoic acid activates transcription, whilst agonism of both dimer partners has an additive effect on activation. Assessment of RXR isoforms in the cell lines used in the current study demonstrated differential expression of RXRs in Ishikawa, RL95 and MFE280 cells, which may account for the distinct responses of these cell lines in response to GW3965 treatment (Supplementary Fig. 5). NR2B1 $(\mathrm{RXR} \alpha)$ mRNA expression was greatest in RL95 cells whilst NR2B2 (RXR $\beta)$ was detected in all cell lines. Notably, mRNA expression of NR2B3 (RXR $\gamma$ ) was not detected in RL95 cells but was abundant in MFE280 cells. Whether changes in the constitution of the receptor isoforms that contribute to the LXR:RXR heterodimer affect responses requires further investigation; however, previous studies demonstrate that targeting retinoid signalling may affect proliferation of EC cells. Notably, retinoic acid (RA) signalling via retinoic acid receptor (RAR) and RXR is reported to inhibit Ishikawa cell proliferation by inducing cell cycle arrest (Cheng et al. 2011) and fenretinide, a synthetic derivative of RA, induced apoptosis of Ishikawa cells (Mittal et al. 2014). These results suggest targeting LXR-dependent signalling with LXR and/or RXR agonists could inhibit proliferation in EC and cancer progression.

Changes in the local inflammatory environment that occur during development and progression of EC may also increase exposure to $27 \mathrm{HC}$ due to infiltration of inflammatory cells. We have previously demonstrated that infiltration of immune cells is increased in EC tissues compared to controls. Notably, the numbers of macrophages, neutrophils and dendritic cells were significantly increased in EC tissues (Wallace et al. 2010) consistent with $27 \mathrm{HC}$-dependent increases in migration of bone marrow-derived $\mathrm{CD}_{11 b^{+}}$cells reported in in vitro assays (Raccosta et al. 2013). In addition, $27 \mathrm{HC}$ increases secretion of CCL2 from macrophages which enhances recruitment of monocytes (Kim et al. 2013) and can also upregulate ER-dependent expression of proinflammatory genes (Umetani et al. 2014). Notably, as Cyp27a1 is reported to be abundant in macrophages (2), these cells may also contribute to an increase in $27 \mathrm{HC}$ within the tumour microenvironment. In support of this idea, increased $27 \mathrm{HC}$ concentrations have been reported in breast cancer tumours (Wu et al. 2013) and increased concentrations of cholesterol have been reported in tumours of various cancer types although they have not been directly measured in EC. 27HC can also promote secretion of TNFA and IL6 from macrophages and TNFA is reported to increase proliferation of human endometrial glandular epithelial cells (Nair et al. 2013). Thus, although in the current study we only investigated the direct impact of $27 \mathrm{HC}$ on proliferation of EC epithelial cells, 27HC may also exacerbate changes within the tissue microenvironment by modulating inflammatory responses, and this merits further investigation in animal models.

\section{Summary}

In the current study, we provide the first evidence to support a mechanistic link between exposure to elevated cholesterol, biosynthesis of $27 \mathrm{HC}$ and EC. Analysis of human stage 1 endometrial adenocarcinomas revealed expression of the key metabolising enzymes of $27 \mathrm{HC}$ was altered in EC consistent with increased exposure to $27 \mathrm{HC}$ as EC progresses from well to poorly differentiated. Although survival rates for EC are high, incidence rates are increasing in line with rates of obesity and a rising incidence in pre- and peri-menopausal women creates unique therapeutic challenges. Based on our novel findings,

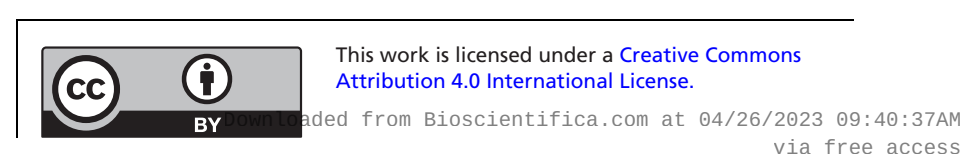


we propose that exposure to $27 \mathrm{HC}$ may influence disease development/progression by activating ER-dependent pathways to increase epithelial cell proliferation. These results suggest strategies that seek to limit exposure to 27HC through lifestyle modification, lipid-lowering drugs such as statins or novel therapeutics that target 27HC synthesis (CYP27A1 inhibitors) may be effective in reducing endometrial proliferation in women at increased risk of developing EC. Taken together, our novel findings suggest that altered cholesterol metabolism, and aberrant exposure to 27HC, may contribute to the development and/or progression of EC.

\section{Supplementary data}

This is linked to the online version of the paper at https://doi.org/10.1530/ ERC-17-0449.

\section{Declaration of interest}

The authors declare that there is no conflict of interest that could be perceived as prejudicing the impartiality of the research reported.

\section{Funding}

Studies undertaken in the Saunders laboratory were supported by MRC Programme Grant G1100356/1 (P T K S) and CRUK development fund (D $A G$ and $P T K S)$.

\section{Author contribution statement}

Experimental design; D A G, F C and P T K S, experimental procedures; D A G, F C, A E Z and F L C, manuscript preparation; D A G, F C and P T K S.

\section{Acknowledgements}

The authors thank Dr Alistair Williams for histological evaluations of endometrial tissue and Prof. Richard Anderson and clinical support team for collection of tissues. They thank members of PTKS laboratory for technical support. Studies undertaken in the Saunders laboratory were supported by MRC Programme Grant G1100356/1 (P T K S) and CRUK development fund (D A G and P T K S). The authors have no conflicts of interest to disclose.

\section{References}

Bombail V, Gibson DA, Collins F, MacPherson S, Critchley HO \& Saunders PT 2010 A role for the orphan nuclear receptor estrogenrelated receptor alpha in endometrial stromal cell decidualization and expression of genes implicated in energy metabolism. Journal of Clinical Endocrinology and Metabolism 95 E224-E228. (https://doi. org/10.1210/jc.2010-0154)

Burkard I, von Eckardstein A, Waeber G, Vollenweider P \& Rentsch KM 2007 Lipoprotein distribution and biological variation of 24S- and 27-hydroxycholesterol in healthy volunteers. Atherosclerosis 194 71-78. (https://doi.org/10.1016/j.atherosclerosis.2006.09.026)
Calle EE \& Kaaks R 2004 Overweight, obesity and cancer: epidemiological evidence and proposed mechanisms. Nature Reviews Cancer 4 579-591. (https://doi.org/10.1038/nrc1408)

Cheng YH, Utsunomiya H, Pavone ME, Yin P \& Bulun SE 2011 Retinoic acid inhibits endometrial cancer cell growth via multiple genomic mechanisms. Journal of Molecular Endocrinology 46 139-153. (https:// doi.org/10.1530/JME-10-0064)

Collins F, MacPherson S, Brown P, Bombail V, Williams AR, Anderson RA, Jabbour HN \& Saunders PT 2009 Expression of oestrogen receptors, ERalpha, ERbeta, and ERbeta variants, in endometrial cancers and evidence that prostaglandin $\mathrm{F}$ may play a role in regulating expression of ERalpha. BMC Cancer 9 330. (https:// doi.org/10.1186/1471-2407-9-330)

DuSell CD, Umetani M, Shaul PW, Mangelsdorf DJ \& McDonnell DP 2008 27-Hydroxycholesterol is an endogenous selective estrogen receptor modulator. Molecular Endocrinology 22 65-77. (https://doi. org/10.1210/me.2007-0383)

Frasor J, Barnett DH, Danes JM, Hess R, Parlow AF \& Katzenellenbogen BS 2003 Response-specific and ligand dosedependent modulation of estrogen receptor (ER) alpha activity by ERbeta in the uterus. Endocrinology 144 3159-3166. (https://doi org/10.1210/en.2002-0143)

Fukunaka K, Saito T, Wataba K, Ashihara K, Ito E \& Kudo R 2001 Changes in expression and subcellular localization of nuclear retinoic acid receptors in human endometrial epithelium during the menstrual cycle. Molecular Human Reproduction 7 437-446. (https:// doi.org/10.1093/molehr/7.5.437)

Gong TT, Li D, Wu QJ \& Wang YZ 2016 Cholesterol consumption and risk of endometrial cancer: a systematic review and dose-response meta-analysis of observational studies. Oncotarget 7 16996-17008. (https://doi.org/10.18632/oncotarget.13796)

Greaves E, Collins F, Critchley HO \& Saunders PT 2013 ERbetadependent effects on uterine endothelial cells are cell specific and mediated via Sp1. Human Reproduction 28 2490-2501. (https://doi. org/10.1093/humrep/det235)

He S \& Nelson ER 2017 27-Hydroxycholesterol, an endogenous selective estrogen receptor modulator. Maturitas 104 29-35. (https://doi. org/10.1016/j.maturitas.2017.07.014)

Johnson SM, Maleki-Dizaji M, Styles JA \& White IN 2007 Ishikawa cells exhibit differential gene expression profiles in response to oestradiol or 4-hydroxytamoxifen. Endocrine-Related Cancer 14 337-350. (https://doi.org/10.1677/ERC-06-0085)

Kim KH, Lee GY, Kim JI, Ham M, Won Lee J \& Kim JB 2010 Inhibitory effect of LXR activation on cell proliferation and cell cycle progression through lipogenic activity. Journal of Lipid Research $\mathbf{5 1}$ 3425-3433. (https://doi.org/10.1194/jlr.M007989)

Kim SM, Lee SA, Kim BY, Bae SS, Eo SK \& Kim K 2013 27-Hydroxycholesterol induces recruitment of monocytic cells by enhancing CCL2 production. Biochemical and Biophysical Research Communications 442 159-164. (https://doi.org/10.1016/j. bbrc.2013.11.052)

Lau K-M, LaSpina M, Long J \& Ho S-M 2000 Expression of estrogen receptor (ER)- $\alpha$ and ER- $\beta$ in normal and malignant prostatic epithelial cells: regulation by methylation and involvement in growth regulation. Cancer Research 60 3175-3182.

Li S, Hu R, Wang C, Guo F, Li X \& Wang S 2014 miR-22 inhibits proliferation and invasion in estrogen receptor alpha-positive endometrial endometrioid carcinomas cells. Molecular Medicine Reports 9 2393-2399. (https://doi.org/10.3892/mmr.2014.2123)

Lindemann K, Vatten LJ, Ellstrom-Engh M \& Eskild A 2009 Serum lipids and endometrial cancer risk: results from the HUNT-II study. International Journal of Cancer 124 2938-2941. (https://doi. org/10.1002/ijc.24285)

Lubahn DB, Moyer JS, Golding TS, Couse JF, Korach KS \& Smithies O 1993 Alteration of reproductive function but not prenatal sexual development after insertional disruption of the mouse estrogen 
receptor gene. PNAS 90 11162-11166. (https://doi.org/10.1073/ pnas.90.23.11162)

Mittal N, Malpani S, Dyson M, Ono M, Coon JS, Kim JJ, Schink JC, Bulun SE \& Pavone ME 2014 Fenretinide: a novel treatment for endometrial cancer. PLoS ONE 9 e110410. (https://doi.org/10.1371/ journal.pone.0110410)

Mouzat K, Prod'homme M, Volle DH, Sion B, Dechelotte P, Gauthier K, Vanacker JM \& Lobaccaro JM 2007 Oxysterol nuclear receptor LXRbeta regulates cholesterol homeostasis and contractile function in mouse uterus. Journal of Biological Chemistry 282 4693-4701. (https://doi.org/10.1074/jbc.M606718200)

Nair S, Nguyen H, Salama S \& Al-Hendy A 2013 Obesity and the endometrium: adipocyte-secreted proinflammatory TNF alpha cytokine enhances the proliferation of human endometrial glandular cells. Obstetrics and Gynecology International 2013 368543. (https:// doi.org/10.1155/2013/368543)

Nelson ER, Wardell SE, Jasper JS, Park S, Suchindran S \& Howe MK 2013 27-Hydroxycholesterol links hypercholesterolemia and breast cancer pathophysiology. Science 342 1094-1098. (https://doi.org/10.1126/ science.1241908)

Nickkho-Amiry M, McVey R \& Holland C 2012 Peroxisome proliferatoractivated receptors modulate proliferation and angiogenesis in human endometrial carcinoma. Molecular Cancer Research 10 441-453. (https://doi.org/10.1158/1541-7786.MCR-11-0233)

Nishida M, Kasahara K, Kaneko M, Iwasaki H \& Hayashi K 1985 Establishment of a new human endometrial adenocarcinoma cell line, Ishikawa cells, containing estrogen and progesterone receptors. Nihon Sanka Fujinka Gakkai Zasshi 37 1103-1111.

Onstad MA, Schmandt RE \& Lu KH 2016 Addressing the role of obesity in endometrial cancer risk, prevention, and treatment. Journal of Clinical Oncology 34 4225-4230. (https://doi.org/10.1200/ JCO.2016.69.4638)

Raccosta L, Fontana R, Maggioni D, Lanterna C, Villablanca EJ, Paniccia A, Musumeci A, Chiricozzi E, Trincavelli ML, Daniele S, et al. 2013 The oxysterol-CXCR2 axis plays a key role in the recruitment of tumor-promoting neutrophils. Journal of Experimental Medicine $\mathbf{2 1 0}$ 1711-1728. (https://doi.org/10.1084/jem.20130440)

Raza S, Meyer M, Goodyear C, Hammer KDP, Guo B \& Ghribi O 2017 The cholesterol metabolite 27-hydroxycholesterol stimulates cell proliferation via ER $\beta$ in prostate cancer cells. Cancer Cell International 17 52. (https://doi.org/10.1186/s12935-017-0422-x)
Sanderson PA, Critchley HO, Williams AR, Arends MJ \& Saunders PT 2017 New concepts for an old problem: the diagnosis of endometrial hyperplasia. Human Reproduction Update 23 232-254. (https://doi. org/10.1093/humupd/dmw042)

Trabert B, Wentzensen N, Felix AS, Yang HP, Sherman ME \& Brinton LA 2015 Metabolic syndrome and risk of endometrial cancer in the United States: a study in the SEER-Medicare linked database. Cancer Epidemiology, Biomarkers and Prevention 24 261-267. (https://doi. org/10.1158/1055-9965.EPI-14-0923)

Umetani M, Domoto H, Gormley AK, Yuhanna IS, Cummins CL \& Javitt NB 2007 27-Hydroxycholesterol is an endogenous SERM that inhibits the cardiovascular effects of estrogen. Nature Medicine $\mathbf{1 3}$ 1185-1192. (https://doi.org/10.1038/nm1641)

Umetani M, Ghosh P, Ishikawa T, Umetani J, Ahmed M, Mineo C \& Shaul PW 2014 The cholesterol metabolite 27-hydroxycholesterol promotes atherosclerosis via proinflammatory processes mediated by estrogen receptor alpha. Cell Metabolism 20 172-182. (https://doi. org/10.1016/j.cmet.2014.05.013)

Vedin L-L, Lewandowski SA, Parini P, Gustafsson J-Å \& Steffensen KR 2009 The oxysterol receptor LXR inhibits proliferation of human breast cancer cells. Carcinogenesis 30 575-579. (https://doi. org/10.1093/carcin/bgp029)

Wallace AE, Gibson DA, Saunders PT \& Jabbour HN 2010 Inflammatory events in endometrial adenocarcinoma. Journal of Endocrinology 206 141-157. (https://doi.org/10.1677/JOE-10-0072)

Way DL, Grosso DS, Davis JR, Surwit EA \& Christian CD 1983 Characterization of a new human endometrial carcinoma (RL95-2) established in tissue culture. In Vitro 19 147-158. (https://doi. org/10.1007/BF02618053)

Wu Q, Ishikawa T, Sirianni R, Tang H, McDonald JG, Yuhanna IS, Thompson B, Girard L, Mineo C, Brekken RA,et al. 2013 27-Hydroxycholesterol promotes cell-autonomous, ER-positive breast cancer growth. Cell Reports 5 637-645. (https://doi.org/10.1016/j. celrep.2013.10.006)

Yang JZ, O'Flatharta C, Harvey BJ \& Thomas W 2008 Membrane ER $\alpha$ dependent activation of PKC $\alpha$ in endometrial cancer cells by estradiol. Steroids 73 1110-1122. (https://doi.org/10.1016/j. steroids.2008.04.012)

Zhao H, Zhou L, Shangguan AJ \& Bulun SE 2016 Aromatase expression and regulation in breast and endometrial cancer. Journal of Molecular Endocrinology 57 R19-R33. (https://doi.org/10.1530/JME-15-0310)

Received in final form 19 January 2018

Accepted 25 January 2018

Accepted Preprint published online 25 January 2018
(C) 2018 The authors Published by Bioscientifica Ltd. Printed in Great Britain
This work is licensed under a Creative Commons Attribution 4.0 International License.

ded from Bioscientifica.com at 04/26/2023 09:40:37AM 